\title{
Quartz wires versus Carbon fibres for improved beam handling capacity of the LEP Wire Scanners
}

\author{
C. Fischer, R. Jung, J. Koopman \\ CERN, SL Division, CH1211 Geneva 23, Switzerland
}

\begin{abstract}
After the first investigations performed in 1994, the study of thermal effects on Carbon and Quartz wires has been pursued in 1995. Carbon wires of $8 \mu \mathrm{m}$ have been studied. Light emission resulting from the two heating mechanisms, electromagnetic fields and collision losses with the beam, were observed. Quartz wires of 10 and $30 \mu \mathrm{m}$ were investigated and light emission due to the heating by collision with the beam was observed. The heat pattern differs completely from that of Carbon fibres. The Quartz wires withstood at $20 \mathrm{GeV}$ circulating currents of at least $8 \mathrm{~mA}$, the 1995 operational level in LEP. Quantitative evaluations and the influence of various dissipation processes are presented with the aim of evaluating a beam current limit.
\end{abstract}

\section{INTRODUCTION}

Investigations made in 1994 with the LEP wire scanners have shown that the major heating mechanism in a conducting wire comes from the electromagnetic fields (1). The effects are felt well before the lepton beam traverses the wire and outside the portion of the wire crossed by the beam. These observations led to the use of quartz, an isolating material, which is only sensitive to energy deposition by collision with the beam and hence withstands higher currents despite a lower fusion point. The comparison between carbon and quartz wires was pursued in 1995 with new CCD cameras.

\section{TESTS DESCRIPTION}

Two multi-fork tanks were installed in 1995 and were equipped with three horizontal and two vertical mechanisms. The forks were made of ceramics and could be observed with CCD cameras through viewing ports (1). These multi-fork tanks 
included the operational vertical profile wire which was made of $30 \mu \mathrm{m}$ Quartz. The three horizontal mechanisms were redundant and were used for material studies. One ceramic fork was fitted with two wires, and during the first part of the year two $8 \mu \mathrm{m}$ carbon wires with $12 \mathrm{~mm}$ spacing were used. This set-up was intended to evaluate very low diameter fibres, so as to limit the effect of beam blowup. One of the two wires broke at the top early in the period under unrecorded conditions, hence only one wire remained operational. Scans were performed during LEP fills at $20 \mathrm{GeV}$ with beam currents going from 0.5 to $7.5 \mathrm{~mA}$ in $1 \mathrm{~mA}$ increments. During a scan, the light emitted from the wire was memorised on a video recorder, i.e. at regular intervals of $20 \mathrm{~ms}$. The second wire broke during a scan with a total beam current of $6 \mathrm{~mA}$ soon after a scan performed at $5 \mathrm{~mA}$. Both wires broke at the top as seen on the recorded light patterns.

The carbon wires were replaced by two quartz wires of 10 and $30 \mu \mathrm{m}$ with the same $12 \mathrm{~mm}$ spacing, and the same tests were resumed. In this case, scans were made up to the operational beam current limit of LEP which was around $8 \mathrm{~mA}$. From light emission it could be observed that the $10 \mu \mathrm{m}$ wire broke probably from fatigue or wearing out after having undergone about 10 scans with a circulating current of $7.3 \mathrm{~mA}$. The thicker $30 \mu \mathrm{m}$ wire survived and was used for further tests. At the end of the run, the wires were inspected and it appeared that the $10 \mu \mathrm{m}$ half wires had been bent towards the extremities of the $30 \mu \mathrm{m}$ wire. This wire was still intact, but the centre was thinned down to a few microns over the beam height and the melted material had collected in a quartz pearl just below.

This confirms clearly the very different behaviour of Carbon and Quartz wires.

\section{CARBON LIGHT EMISSION PATTERN}

The digitised video recording of the light emitted by the $8 \mu \mathrm{m}$ carbon wire when scanning horizontally a $0.8 \mathrm{~mA}$ beam is shown in Figure 1 . The wire lights up to the points where it is fixed to the ceramic fork. Hence the illuminated length is a precise indication of the total wire length $(29 \mathrm{~mm})$. The time dependence of the radiation pattern is given by the time structure of the video signal, i.e. $20 \mathrm{~ms}$ per profile. This time corresponds to a fork displacement of $5 \mathrm{~mm}$ at a wire speed of $0.25 \mathrm{~m} / \mathrm{s}$. The two large hot areas at the right and left sides of each profile are characteristic of the heating of the wire under the influence of electromagnetic fields. They correspond to portions of the wire which are not traversed by the beam and appear well before the wire crosses the beam. This heating is induced by the vertical component of the electric field, direct and wakefield, generated by the passage of the 50 ps (r.m.s.) short bunches. The effect of collision losses is only observed on one curve which exhibits a reduced intensity central spot with a width of about $2 \mathrm{~mm}$. Its amplitude is lower by more than a factor of 5 with respect to the two radiating ends. The two adjacent curves have not this central spot, indi- 
cating that within $20 \mathrm{~ms}$ the temperature has decreased below the sensitivity threshold of the camera.

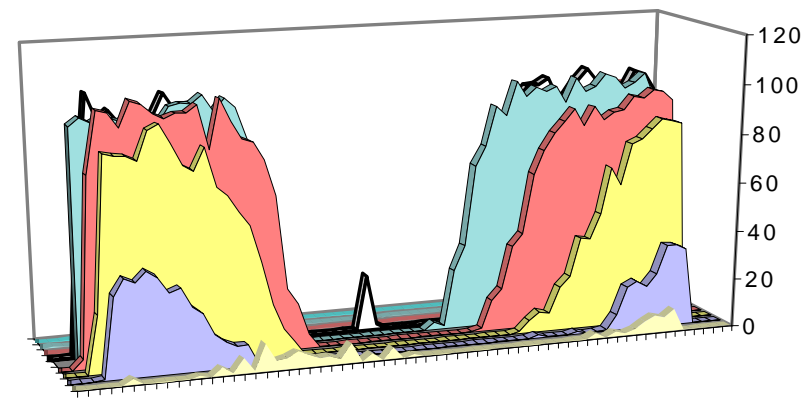

FIGURE 1: Digitised video recording of an $8 \mu \mathrm{m}$ carbon wire scanning a $0.8 \mathrm{~mA}$ beam. The wire is parallel to the horizontal axis, and the light intensity is plotted along the vertical axis (arbitrary units). Successive profiles are separated by $20 \mathrm{~ms}$. The central spot corresponds to the passage of the wire through the beam.

The temperature of this spot cannot be scaled with that of the extremities which are well above the saturation level of the camera. The light emission is stronger on the second half of the scan, after beam traversal as the wire is heating up regularly. The level of radiation of the wire extremities and their length increase with beam current. The structure of the wakefields is clearly visible at high currents: see Figure 2, made during a sweep at a current of $5 \mathrm{~mA}$.
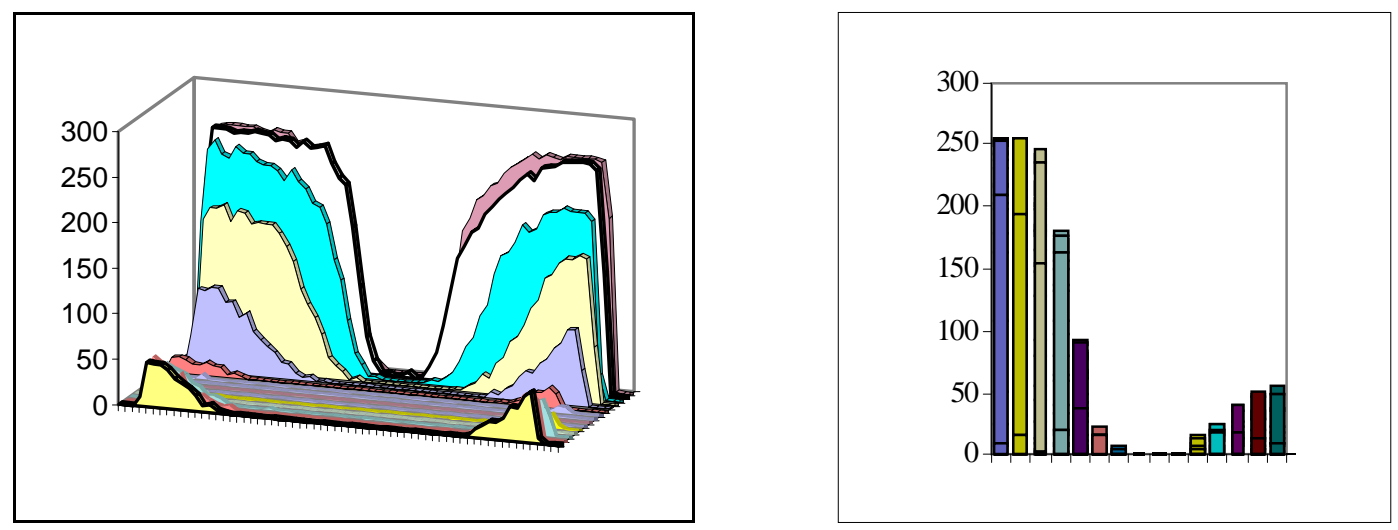

FIGURE 2: Light emission of an $8 \mu \mathrm{m}$ carbon wire scanning a $5 \mathrm{~mA}$ beam. Left: Light intensity pattern along the Carbon wire as a function of its position. Right: Maximum light emission evolution. The wire/beam encounter takes place at the left. The light emission hole indicates an area with vanishing wakefields. 
The central hot spot generated by the beam interaction is barely visible on this scan as the gain was reduced by a factor 100 to limit saturation effects. Wakefield calculations have been made with the program MAFIA to evaluate this type of pattern. The calculations show that the wakefields last for approximately $250 \mathrm{ps}$. The computed voltage differences, see Figure 3, have been used to estimate the temperature pattern along the wire. It is close to the measured one and a maximum temperature of $3300 \mathrm{~K}$ has been found for a beam of $5 \mathrm{~mA}(2)$.

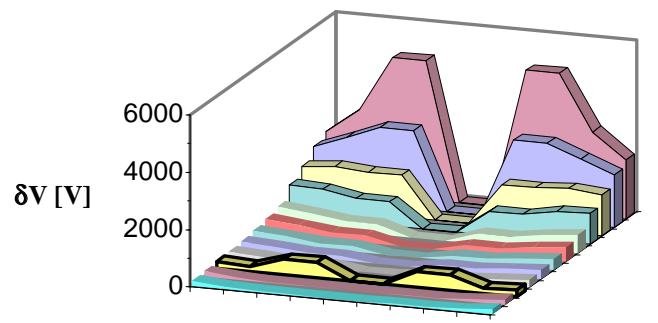

FIGURE 3: Voltage differences calculated along the wire (5 mm steps) as a function of wire position during a scan of 32 bunches with a total intensity of $5 \mathrm{~mA}$ (bunch trains).

The previous observations show clearly that the main heating effect in conducting wires is by the electric component of the wakefields. It explains also why the wires always break towards an extremity. As the vacuum tank has been made as well as possible from a wakefield point of view, it has a loss factor of $0.1 \mathrm{~V} / \mathrm{pC}$, there is little to be gained to minimise the wakefields and to increase the beam handling capacity beyond $6 \mathrm{~mA}$. The only possibility to increase this current limit would be to install the scanner at the crossing point (IP1) where the electric fields of the electron and positron beams would cancel under ideal conditions.

\section{QUARTZ WIRE LIGHT EMISSION}

Quartz wires have been used to replace the carbon fibres, because they are the most easily available non conducting wires with diameters from 5 to $30 \mu \mathrm{m}$. They have a melting point around $1700{ }^{\circ} \mathrm{C}$ and are acceptable from beam blow-up and energy deposition points of view. A typical radiation pattern is given in Figure 4 taken at a circulating beam of $7.3 \mathrm{~mA}$. Only the central region where beam and wire interact is now radiating and nothing is observed before crossing the beam. The first curve corresponds to the spot emitted by the $30 \mu \mathrm{m}$ wire when crossing the beam. The amplitude of the next curves are slightly reduced as the wire starts to cool down. However, $60 \mathrm{~ms}$ later (fourth curve), the amplitude is again increased as the second wire $(10 \mu \mathrm{m})$ starts to cross the beam and its signal adds to the remnant radiation of the first wire. The subsequent curves give an indication of the thermal conduction time constant of the quartz wires. Within 60 to $80 \mathrm{~ms}$ the 
amplitude decreases by a factor 10 . The thermal conduction is a slow process in quartz. The curves have a width of approximately $2 \mathrm{~mm}$ which fits well with the r.m.s. beam size of $800 \mu \mathrm{m}$.
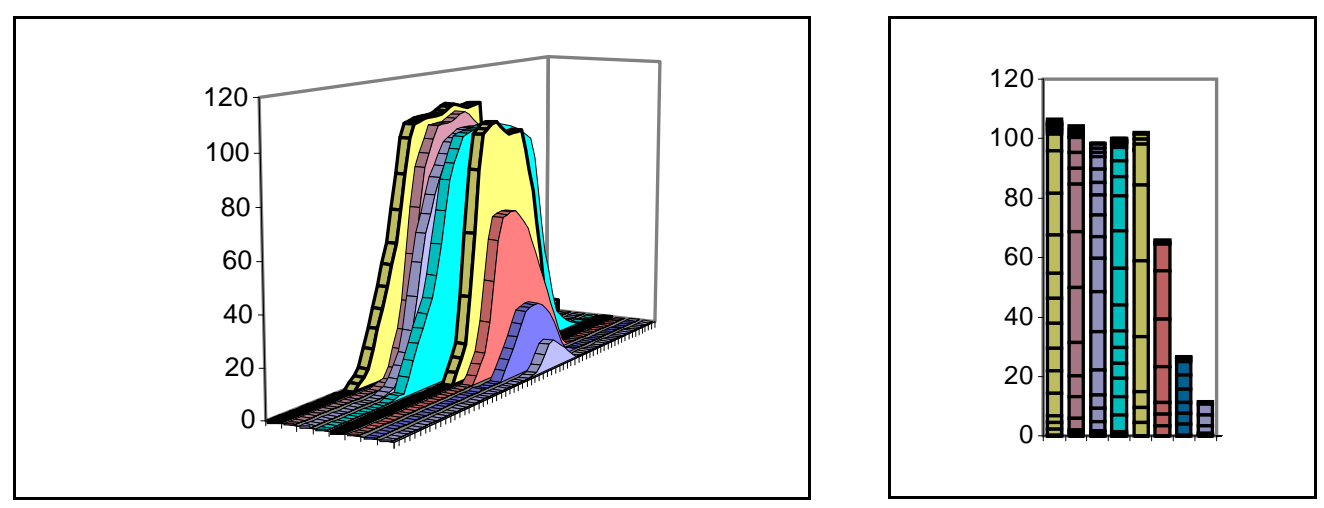

FIGURE 4: Radiation patterns from the two quartz wires (enlarged scale) versus wire position for a $7.3 \mathrm{~mA}$ beam. The first beam encounter is at the left in both graphs.

Right: Maximum light emission evolution, with the two beam encounters clearly visible.

The same pattern is obtained when scanning in the other direction where the 10 $\mu \mathrm{m}$ wire is the first to encounter the beam. Its emission characteristics, in width and amplitude, are very similar, which corroborates the calculations performed in the next sections.

\section{HEATING FROM COLLISION LOSSES}

Detailed calculations can be found in (2). The basic features are recalled here. The ionisation losses for high energy electrons are given by (3):

$$
\frac{1}{\rho} \frac{d E}{d x}=\frac{0.153}{\beta^{2}} \frac{Z}{A}(F(\beta)-2 \ln I-\delta(X)) \quad\left[\mathrm{MeV} \mathrm{g}^{-1} \mathrm{~cm}^{2}\right]
$$

where $\delta(X)$ describes the density effect of the medium and is given by:

$\delta(X)=4.605 X+C \quad$ with $X=\log (\beta \gamma)$ and $C$ to be found in Table 1 . The density effect decreases the ionisation losses by some $36 \%$ in both materials. The energy lost by a beam of $\mathrm{N}$ particles during a scan is:

$$
\Delta \mathrm{E}(\text { scan })=\mathrm{N}<\mathrm{x}>\mathrm{dE} / \mathrm{dx}
$$

with $\langle x\rangle$ the average wire thickness seen by each particle during a scan (2):

$$
<\mathrm{x}>=\frac{\pi R^{2}}{v T}
$$

where $\mathrm{R}$ is the wire radius, $\mathrm{v}$ the wire speed $(0.25 \mathrm{~m} / \mathrm{s})$, and $\mathrm{T}$ the beam revolution period $(88.9 \mu \mathrm{s}$ in LEP). 
TABLE 1: Comparative data for Carbon and Quartz wires for a $7.5 \mathrm{~mA}$ total intensity

\begin{tabular}{|c|c|c|c|}
\hline & Carbon & \multicolumn{2}{|c|}{ Quartz } \\
\hline$\overline{Z / A}$ & 0.4995 & \multicolumn{2}{|c|}{0.4993} \\
\hline $\mathrm{I}[\mathrm{eV}]$ & 78 & \multicolumn{2}{|c|}{139} \\
\hline$\rho\left[\mathrm{g} \mathrm{cm}^{-3}\right]$ & 2.26 & \multicolumn{2}{|c|}{2.2} \\
\hline$c_{p}\left[\mathrm{~J} \mathrm{~g}^{-1} \mathrm{~K}^{-1}\right]$ & $1.647^{*}$ & \multicolumn{2}{|c|}{$1.124^{*}$} \\
\hline $\mathrm{k}\left[\mathrm{W} \mathrm{\textrm {m } ^ { - 1 } \mathrm { K } ^ { - 1 } ]}\right.$ & 120 & \multicolumn{2}{|c|}{2.7} \\
\hline Sublimation/Melting point $\left[{ }^{\circ} \mathrm{C}\right]$ & 3700 & \multicolumn{2}{|c|}{1700} \\
\hline C & 2.868 & \multicolumn{2}{|c|}{4.003} \\
\hline$\delta(X)$ & 18.28 & \multicolumn{2}{|c|}{17.15} \\
\hline 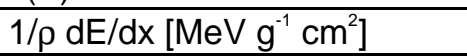 & 2.335 & \multicolumn{2}{|c|}{2.333} \\
\hline $\mathrm{D}[\mu \mathrm{m}]$ & 8 & 10 & 30 \\
\hline$<\mathrm{x}>[\mu \mathrm{m}]$ & 2.3 & 3.5 & 31.8 \\
\hline$\Delta \mathrm{E}$ (scan) [MeV] & $4610^{8}$ & $7010^{8}$ & $63010^{8}$ \\
\hline $\mathrm{W}[\mathrm{MeV}]$ & $28.310^{8}$ & $4210^{8}$ & $37410^{8}$ \\
\hline$\eta_{1}$ & $61 \%$ & $59 \%$ & $59 \%$ \\
\hline$\eta_{2}$ & $75 \%$ & $76 \%$ & $68 \%$ \\
\hline$\eta$ & $54 \%$ & $55 \%$ & $59 \%$ \\
\hline$\Delta \mathbf{T}[\mathrm{K}]$ & 1070 & 1600 & 1700 \\
\hline
\end{tabular}

* averaged from 300 to $1800 \mathrm{~K}$

The fraction of the energy actually deposited in the wire has to be calculated.

The energy deposited between $\mathrm{E}$ and $\mathrm{E}+\mathrm{dE}$ at a depth $\mathrm{x}$ by an incident particle is:

$$
d W=\frac{d^{2} N}{d E d x} E x d E
$$

and between $I$, the binding electron energy, and $E_{\max }$, each particle will deposit:

$$
W=\int_{I}^{E \max } d W
$$

The distribution of secondary electrons with energy $\mathrm{E}$ is given by (4):

$$
\frac{d^{2} N}{d E d x}=0.153 \frac{Z}{A} \frac{z^{2}}{\beta^{2}} \rho \frac{F}{E^{2}}
$$

For $20 \mathrm{GeV}$ leptons, $\mathrm{E}_{\max }=10 \mathrm{GeV}$ and $\mathrm{F}=1$ (4). For $\mathrm{N}$ particles with average path $\langle x\rangle$, one gets for $W$ the values of Table 1 and hence the fraction $\eta_{1}$ of energy transmitted to knock-on electrons. Some knock-on electrons escape from the wire and will not contribute to the wire heating. Their fraction $\eta_{2}$ is :

$$
\eta_{2}=\frac{\ln \left(E_{\max } /<E i>\right)}{\ln \left(E_{\max } / I\right)}
$$

where $\langle\mathrm{Ei}\rangle$ is the average ionisation threshold:

$$
<\operatorname{Ei}>=\frac{1}{1.296}(\rho<x>)^{0.58}
$$


Finally, the energy actually deposited inside the wire is: $E_{d}=\Delta E(\operatorname{scan}) \eta$, with $\eta$ the overall wire heating efficiency given by:

$$
\eta=\left(1-\eta_{1}\right)+\left(1-\eta_{2}\right) \eta_{1}
$$

varying from 54 to $59 \%$ for the different wires, see Table 1.

If $\sigma_{\mathrm{v}}$ is the r.m.s. vertical beam dimension $(800 \mu \mathrm{m}$ at $20 \mathrm{GeV})$, the wire volume $\mathrm{V}$ heated during a horizontal scan is:

$$
\mathrm{V}=\pi \mathrm{R}^{2} \sqrt{2 \pi} \sigma_{\mathrm{v}}
$$

and if $c_{p}$ is the heat capacity averaged over $1500^{\circ}$, then the temperature rise is:

$$
\Delta \mathrm{T}=\mathrm{E}_{\mathrm{d}} /\left(\mathrm{V} \rho \mathrm{c}_{\mathrm{p}}\right)
$$

The final results are given in Table 1 and show temperature increases of $1100^{\circ}$ for carbon and 1600 to $1700^{\circ}$ for quartz. They confirm that losses by collision are large enough to bring the quartz wires close to their fusion point, but will not destroy the carbon fibres.

\section{EFFECT OF THERMAL CONDUCTION}

The thermal time constant in the wire has been estimated with a simple model.

The wire section length which is heated by the beam is:

$$
\mathrm{L}=\sqrt{2 \pi} \sigma_{\mathrm{v}}
$$

Considering one half of the wire starting at its centre (hot point), the average path along this section is $\mathrm{L} / 4$ and hence its thermal time constant $\tau$ is:

$$
\tau=\mathrm{R}_{\mathrm{th}} \mathrm{C}_{\mathrm{th}}=\mathrm{c}_{\mathrm{p}} \rho(\mathrm{L} / 4)^{2} / \mathrm{k}
$$

where $\mathrm{k}$ is the thermal conductivity (Table 1 ). The results are: $\tau=15 \mathrm{~ms}$ for carbon and $500 \mathrm{~ms}$ for quartz. In carbon, $\tau=15 \mathrm{~ms}$ explains why the central spot disappears within consecutive video acquisitions. For quartz, the value of $\tau$ corroborates also the observations. It leads to a temperature decrease of $15 \%$ within 80 ms which in turn corresponds to a reduction of the emitted power by a factor of 5 in the CCD spectrum according to Planck's law. During a complete wire sweep through the beam (typically $7 \mathrm{~ms}$ ) the loss by thermal conduction in quartz is about $1 \%$ of the deposited energy and will not induce any beneficial cooling.

\section{EFFECT OF RADIATION LOSSES IN QUARTZ}

From Stephan-Boltzmann's law, the total emitted power is $\mathrm{P}=\varepsilon \sigma \mathrm{A} \mathrm{T}^{4}$ with $\sigma=5.67210^{-8} \mathrm{~W} \mathrm{~m}^{-2} \mathrm{~K}^{-4}$, the radiating area $\mathrm{A}=\pi \mathrm{D} \mathrm{L}$ and the quartz emissivity $\varepsilon=0.9$ at room temperature. At $1700 \mathrm{~K}$ one finds: $\mathrm{P}=0.03 \mathrm{~W}$ and $\mathrm{P}=0.08 \mathrm{~W}$ for the 10 and the $30 \mu \mathrm{m}$ quartz wires. Assuming the emission to take place mainly in 
the second half of the beam traversal (high temperature), it will subtract 17 and $4.5 \%$ from the energy absorbed during a scan.

Thermo-ionic emission can be neglected below $2000 \mathrm{~K}$, i.e. for quartz.

\section{CONCLUSION}

Thermal effects generated in the LEP wire scanners by electromagnetic fields have been observed directly and quantified for conducting materials such as carbon. They lead to temperature increases four times higher than losses by collisions for a beam of $5 \mathrm{~mA}$ and are the major source of wire heating. Non conducting wires are not affected by them and quartz wires withstood beam currents up to 8 $\mathrm{mA}$. Both the calculations of energy deposition by collision with the beam and the inspection of the wires confirm that this current level is close to the possible operational threshold at $20 \mathrm{GeV}$. This threshold has to be reduced for horizontal scans at higher energies because the vertical beam emittances will be much smaller. The experience gained with quartz wires indicates a safe operational beam current level close to $5 \mathrm{~mA}$ at the LEP 2 energy. Tests will be pursued in 1996 with quartz to confirm this limit and also with $15 \mu \mathrm{m} \mathrm{SiC}$ wires. The use of diamond wires is also considered and the installation of a horizontal wire scanner at IP1 will be evaluated.

\section{ACKNOWLEDGEMENTS}

The ingenuity of J. Camas in handling and installing all kinds of fibres is much appreciated. P. Valentin performed together with A. Wagner the calculations with MAFIA and their help was precious. We also appreciate the collaboration of our colleagues of the Vacuum Group for the recurrent installation work.

It is a pleasure to acknowledge their contribution in the completion of this study.

\section{REFERENCES}

1. J. Camas et al.: Observation of thermal effects on the LEP wire scanners, CERN SL/95-20 (BI), May 1995, and Proc. of the 1995 IEEE PAC, Dallas

2. C. Fischer, P. Valentin: An Evaluation of Various Heating Mechanisms in the LEP Wire Scanners, Note to be published

3. R.M. Sternheimer, M.J.Berger, S.M. Seltzer: Atomic Data and Nuclear Data Tables 30, 261271 (1984)

4. Review of Particles Properties, Phys. Rev. D, Vol 45, N 11, June 1992 